\title{
Femmes et expériences de la vie avec le VIH/sida
}

\author{
Janine PIERRET ${ }^{1}$
}

\section{Résumé}

Trois types d'expériences de la vie avec le VIH/sida - continuité, retournement et enfermement - ont été construits à partir de l'analyse d'entretiens qualitatifs recueillis entre 1990 et 2000 . Cette typologie a permis de prendre en compte les effets conjugués de la durée et des changements médicaux liés à l'apparition des traitements antirétroviraux hautement actifs, en 1996. Elle rend compte de ce que les personnes infectées entendent par « vivre normalement ». La situation des femmes qui vivent avec le virus présente des spécificités, en particulier au niveau du rapport au corps et de la vie sexuelle et affective.

Mots clés : femmes, types d'expérience, vie avec le VIH, vie normale, temporalité, médecine.

Au début des années 1990, R Weitz soulignait l'absence de pouvoir d'action (powerlessness) et l'invisibilité des femmes infectées par le VIH, qui étaient également peu nombreuses dans les enquêtes qualitatives [1-3]. À partir de 1995-1996, des recherches en sciences sociales ont porté spécifiquement sur les femmes pour pallier cette absence et souligner leurs difficultés à s'insérer dans un discours sur une maladie qui concernait majoritairement des hommes [4-7]. Cet article cherche à comprendre l'expérience de femmes vivant avec le VIH et à en souligner la spécificité en regard de celle des hommes, à partir d'une typologie des expériences de la vie avec le VIH [8]. Les trois types d'expérience, construits pour rendre compte des effets conjugués de la durée - entre 1990 et 2000 - et des changements dans le contexte médical depuis l'apparition des traitements en 1996, servent de cadre à cette analyse.

Depuis le début des années 2000, les caractéristiques épidémiologiques du VIH/sida se sont modifiées, avec une augmentation du nombre de transmissions hétérosexuelles et de femmes originaires des zones endémiques, Caraïbes et pays d'Afrique sub-saharienne. Ce texte ne permet pas de comprendre les problèmes spécifiques de ces femmes récemment diagnostiquées, mais il éclaire ce que signifie vivre avec le VIH pour des femmes infectées par relations hétérosexuelles, injection de drogue et de sang contaminé, et non entrées dans la maladie sida.

CNRS, UMR 8169, CERMES, Villejuif.

\section{Méthodologie et cadre d'analyse}

Trois enquêtes qualitatives ont eu lieu en 1990, 1996 et 19982000 auprès de 118 personnes vivant avec le VIH, et 167 entretiens en profondeur ont été recueillis. Chacune des enquêtes s'est déroulée selon un protocole de recherche semblable (mode d'accès et de sélection des personnes, dispositif de recueil des données) pour répondre à la même question : comment vit-on quand on est atteint d'une maladie mortelle dans un délai plus ou moins rapide? La consigne de départ des entretiens, largement ouverte, se présentait sous une forme unifiée et de la façon suivante : j'aimerais que vous me parliez, que vous me racontiez comment on vit... Chaque personne abordait spontanément et dans l'ordre qu'elle souhaitait les différents aspects de sa vie, avec peu d'interventions de l'enquêtrice. D'une durée moyenne supérieure à une heure, tous les entretiens ont été enregistrés et intégralement retranscrits.

En 1990, l'enquête qualitative par entretiens en profondeur a porté sur 53 hommes séropositifs et infectés depuis plus de deux ans par des produits anti-hémophiliques et relations sexuelles. En 1996, l'enquête sociologique a été associée à un protocole de recherche biomédical appelé ALT (Asymptomatiques à long terme), mis en place en 1994 par l'Agence nationale de recherches sur le sida et les hépatites virales (Anrs). Trente personnes dont six femmes infectées par le VIH depuis plus de huit ans, sans traitement et non malades ont accepté de participer à l'enquête qualitative. Celle-ci cherchait, sur le même modèle que la précédente, à analyser la façon dont se vit une longue durée de contamination par le VIH et les aménagements éventuels au cours du temps que cette situation implique.

Entre janvier 1998 et juillet 2000, dans un contexte bouleversé par l'apparition des traitements antirétroviraux hautement actifs, une enquête qualitative a été menée au sein de la cohorte nationale Anrs-Aproco (AntiPROtéases Cohorte). Son objectif était d'analyser la place et le sens du traitement dans la vie quotidienne de personnes recevant pour la première fois ce type de traitement. Trente-cinq personnes volontaires dont huit femmes ont été interviewées. Le protocole de recherche prévoyait trois entretiens : au cours du premier, du huitième et du vingtième mois de traitement [9]. Quatre femmes ont été interviewées trois fois, deux femmes l'ont été deux fois et deux, seulement la première fois.

Les 167 entretiens recueillis entre 1990 et 2000 ont fait l'objet d'une nouvelle analyse pour prendre en compte les effets de 
Tableau 1

Caractéristiques des femmes interviewées au moment de l'entretien

ALT (1996-1997)

\begin{tabular}{lllcllcc}
\hline \multicolumn{1}{c}{ Prénoms } & Âge & $\begin{array}{c}\text { Mode de } \\
\text { contamination }\end{array}$ & $\begin{array}{c}\text { Année de } \\
\text { découverte }\end{array}$ & $\begin{array}{c}\text { Niveau } \\
\text { d'éducation }\end{array}$ & Emploi & $\begin{array}{c}\text { Situation } \\
\text { affective }\end{array}$ & $\begin{array}{c}\text { Enfants } \\
\text { Région de } \\
\text { résidence }\end{array}$ \\
\hline Jeanne & 33 ans & hétérosexuel & 1986 & secondaire & infirmière & couple & Est \\
\hline Viviane & 39 ans & hétérosexuel & 1985 & primaire & employée & seule et veuve & 1 \\
\hline Sophie & 33 ans & drogue & 1986 & secondaire & graphiste & couple & Sud \\
\hline Geneviève & 36 ans & hétérosexuel & 1987 & secondaire & employée & seule & veuve et couple de France \\
\hline Cécile & 39 ans & drogue & 1986 & secondaire & AAH & Ouest \\
\hline Christiane & 42 ans & transfusion & 1985 & supérieur & médecin & couple & Île de France \\
\hline
\end{tabular}

Aproco (1998-2000)

\begin{tabular}{|c|c|c|c|c|c|c|c|c|c|c|}
\hline Prénoms & Âge & $\begin{array}{c}\text { Mode de } \\
\text { contamination }\end{array}$ & $\begin{array}{l}\text { Année de } \\
\text { découverte }\end{array}$ & $\begin{array}{c}\text { Niveau } \\
\text { d'éducation }\end{array}$ & Emploi & $\begin{array}{l}\text { Situation } \\
\text { affective }\end{array}$ & Enfants & $\begin{array}{l}\text { Région de } \\
\text { résidence }\end{array}$ & $\begin{array}{c}\text { Traitement } \\
\text { avant Aproco }\end{array}$ & $\begin{array}{c}\text { Nombre } \\
\text { d'entretiens }\end{array}$ \\
\hline Fatou* & 39 ans & hétérosexuel & 1994 & primaire & artiste & couple & 2 & Ouest & oui & 1 \\
\hline Florence & 38 ans & drogue & 1991 & primaire & $\mathrm{AAH}^{\star \star \star}$ & seule et veuve & 2 & Île-de-France & non & 3 \\
\hline Jacqueline & 38 ans & drogue & 1987 & secondaire & AAH & seule et divorcée & 3 & Sud & oui & 2 \\
\hline Patricia & 39 ans & hétérosexuel & 1992 & secondaire & employée & couple & 2 & Sud & oui & 2 \\
\hline Françoise & 35 ans & drogue & 1987 & secondaire & AAH & seule et divorcée & 1 & Sud & oui & 1 \\
\hline Oriane & 38 ans & drogue & 1985 & primaire & employée & seule et divorcée & 1 & Sud & non & 3 \\
\hline Odile & 27 ans & hétérosexuel & 1996 & primaire & $\begin{array}{l}\text { Chômage et } \\
\text { employée }\end{array}$ & couple & 1 & Sud & non & 3 \\
\hline Nicole & 35 ans & hétérosexuel & 1998 & secondaire & $\begin{array}{l}\text { Employée et } \\
\text { formation }\end{array}$ & $\begin{array}{c}\text { seule et couple } \\
\text { en } 2000\end{array}$ & & Sud & oui & 3 \\
\hline
\end{tabular}

- Seule femme originaire d'Afrique sub-saharienne.

$*$ AAH : Allocation d'adulte handicapé.

la durée et des changements survenus en 1996. Une analyse inductive centrée sur les propos des personnes interviewées a permis d'analyser comment celles-ci disaient avoir organisé et construit leur vie avec le VIH. L'imprégnation par lecture répétée et approfondie de tous les entretiens a mis en évidence la diversité des préoccupations exprimées par les personnes, puis de les organiser autour de grandes thématiques et de dégager des principes structurants.

En 1996, puis entre 1998 et 2000, 14 femmes et 51 hommes ont été interviewés.

L'objectif de cet article est de reprendre les 24 entretiens réalisés avec les 14 femmes pour présenter de façon systématique leur situation et en souligner la spécificité, ce qui n'a pas été fait dans l'analyse générale. Neuf d'entre elles ont découvert leur infection avant 1990, quatre entre 1991 et 1996 et une après 1997. Six ont été contaminées par injection de drogue intraveineuse, une par transfusion sanguine et sept par relations hétérosexuelles. Au moment de l'entretien, sept vivaient en couple (l'une d'entre elles était veuve), dont deux en couples séroconcordants, sept étaient seules dont deux veuves, trois divorcées ou séparées et deux ont toujours vécu seules. Huit femmes ont eu des enfants avant de connaître leur infection, dix exercent une activité professionnelle et quatre reçoivent des prestations sociales (tableau 1)

Le cadre d'analyse centré sur les conséquences de la survenue d'une maladie chronique sur la vie des personnes elles-mêmes est celui de l'expérience de la maladie [10-13]. Il a été mis en œuvre pour étudier, à un moment donné, la diversité de ce que recouvre " vivre avec la maladie ", en portant l'attention sur les rapports entre subjectivité, conditions de vie et facteurs culturels [14]. Mais peu de recherches ont pris en compte l'impact des facteurs structurels [15]. Pourtant les technologies médicales comme les changements dans la politique hospitalière et son organisation ont des répercussions sur la vie des malades $[16,17]$. L'arrivée des trithérapies dans la maladie sida, qui depuis 1982 était synonyme de mort, est considérée comme un facteur structurel. Ce texte propose d'apprécier à la fois l'impact du temps-durée et les 
changements du contexte médico-scientifique et politique sur ce que des personnes infectées par le VIH entendent quand elles disent « vivre normalement ».

\section{Une typologie des expériences de la vie avec le VIH}

Trois types d'expérience de la vie avec le VIH, "continuité sous contraintes", "discontinuité et retournement " et "enfermement » ont été construits à partir de deux dimensions : la forme prise par les entretiens (récit chronologique ou non, rapports passé/présent/futur, changements ou non) et les catégories spontanément évoquées pour faire le récit de l'expérience. La centralité du travail et de l'activité professionnelle, le contrôle de l'information, les rapports avec la médecine et les professionnels de soins, les diverses formes d'investissement dans le couple et la famille ou encore dans des activités personnelles développées depuis l'infection par le VIH sont les catégories spontanément abordées. Présentes au cours des dix années étudiées et dans chacun des types d'expérience, elles ont été analysées comme des ressources qui sont autant des enjeux que des problèmes à résoudre.

Ces trois types connaissent des infléchissements, mais ne sont pas radicalement modifiés au cours de la période étudiée. Ils permettent de comprendre les effets de la durée quand on vit sans symptôme dans un temps incertain avec une infection grave et mortelle pour laquelle la médecine n'a guère eu de moyens d'intervention pendant plusieurs années. Ils prennent aussi leur sens en fonction des conditions objectives de vie et des transformations structurelles survenues pendant la période.

Si les conditions de vie sont relativement diversifiées, les types d'expérience de la vie avec le VIH sont profondément marqués par ces institutions structurantes des identités dans notre société que sont d'un côté la participation à la production et le travail professionnel, et de l'autre l'État-providence avec son classement des individus et l'ouverture de droits [18]. Nous avons montré [19] que la maladie d'aujourd'hui ne pouvait pas se comprendre sans les rapports qu'elle entretient avec ces deux institutions, ainsi qu'avec une troisième, centrale dans notre société, la médecine. La maladie se vit en référence au travail professionnel en termes d'activité ou d'inactivité, dans une relation avec l'institution médicale qui, en l'identifiant, la légitime et peut conduire à l'ouverture de droits dans le cadre de l'État-providence. L'expérience de la maladie est indissociable de ce triptyque institutionnel : activité professionnelle, État-providence et institution médicale.

\section{Continuité sous contraintes}

Ce premier type est organisé autour de la référence normative à la vie avant le VIH et correspond au plus grand nombre de personnes et de femmes interviewées (huit sur quatorze). Elles sont toutes insérées socialement et professionnellement au moment où elles apprennent leur contamination et se demandent " comment vivre maintenant? ". L'image que l'on veut donner de soi et celle que les autres ont de vous ne doit pas connaître de défaillance.

Cette vie normale qu'il s'agit de maintenir avec et malgré l'infection par le VIH s'appuie sur deux pivots qui sont aussi des enjeux : la poursuite du travail et le contrôle de l'information. Ces femmes ont toutes une activité professionnelle qu'elles avaient déjà au moment de la découverte du virus. Plusieurs années ont pu s'écouler - parfois plus de dix ans - sans que pour autant, elles aient modifié leur rapport au travail mêrne si certaines ont changé d'emploi. Odile, qui était au chômage en 1998, a retrouvé un emploi en 2000 et Nicole a atteint, en 2000, son objectif de reconversion professionnelle qu'elle avait entrepris avant la découverte du VIH. Si le travail est essentiel à l'équilibre, il signifie surtout le maintien de la continuité de la vie. "C'est le travail et ses exigences qui continuent à assurer la dignité des individus et à entretenir l'essentiel des échanges sociaux » [20, p. 21]. Ces femmes valorisent d'autant plus l'utilité sociale du travail qu'elles savent que le développement de la "maladie sida ", en les coupant du monde du travail, les coupera du monde social. L'activité professionnelle est à la fois synonyme et condition de "la vie normale ".

Contrôler l'information sur la contamination, savoir à qui, quand et comment en parler est la seconde condition essentielle du maintien de la « vie normale " pour toutes les personnes vivant avec le $\mathrm{VIH}$. Mais pour les femmes correspondant à ce type d'expérience et quel que soit le mode de transmission, le maintien du secret sur l'infection par le VIH est fondamental. Plusieurs d'entre elles ont découvert ou appris " par hasard » qu'elles étaient infectées par le VIH. Vivant une relation de couple suivie et régulière depuis plusieurs années avec un compagnon ou un conjoint, Geneviève, Nicole, Fatou et Patricia n'ont jamais pensé qu'elles pourraient être concernées comme le raconte Geneviève : "J'étais avec un copain pendant un an et demi, on vivait ensemble et, un jour, il a eu la bonne idée d'aller faire un don du sang. Ce que je ne savais pas c'est qu'avant il se droguait. Le courrier est arrivé, il ne vivait plus là depuis pas très longtemps, j'ai ouvert pour voir ». Sans connaissance personnelle dans leur entourage, ces femmes vivaient en dehors de tout rapport avec le $\mathrm{VIH} /$ sida. Cet éloignement du VIH/sida et des personnes atteintes a été plus fréquemment observé chez les femmes et analysé comme un élément d'enfermement dans le silence, la honte et la culpabilité d'une maladie qui a longtemps concerné des hommes et des groupes minoritaires [1, p $104 ; 4$, p $5 ; 7$, p 1735].

Le secret est partagé avec un tout petit nombre de proches (le conjoint, certains membres de la famille restreinte, un ou une ami(e) intime), mais il est toujours strictement maintenu dans le milieu de travail. Et si les raisons de se taire sont le refus de la compassion, la peur de l'incompréhension et du rejet, c'est 
surtout la peur que le regard de l'autre change et le besoin de taire qui dominent et concernent un nombre de proches relativement étendu et diversifié. Christiane et son mari ont fait le choix de taire l'infection dans leur famille et dans leur entourage proche : " J'ai une amie qui est vraiment ma meilleure amie à qui je ne l'ai jamais dit parce que dès qu'elle a un rhume son mari met un masque [...] C'est quelqu'un qui psychologiquement est assez fragile et elle n'aurait jamais pu le cacher et je ne suis pas sûre de la réaction de son mari ». Mais ce secret est un lieu de tensions, à la fois pour la personne et dans ses relations à l'autre ; le maintenir demande une vigilance permanente, car le moindre signe visible, notamment aujourd'hui la prise des médicaments, risque de dévoiler ce que l'on veut cacher. En effet, prendre un traitement quotidiennement et régulièrement conduit Patricia, Fatou, Oriane et Nicole à adopter des techniques de dissimulation, tant dans la vie quotidienne que sur le lieu de travail (mettre les médicaments dans un papier banalisé, se cacher pour les prendre). Patricia " trouve que c'est très dur à prendre, et il faut les prendre une heure avant manger. Alors, ça aussi, si vous êtes invitée chez des amis, on vous invite à l'apéritif à 7 heures $d u$ soir, je viens de prendre mon cachet, je ne peux pas dire : "Je ne peux pas manger, pendant une heure!" Ces cachets quand vous allez chez quelqu'un, c'est pas discret [...] Ma fille me dit : "Mais maman, pourquoi tu prends tous ces médicaments?" Puis, elle fait comme moi maintenant, elle me dit: "Quand tu vas te réveiller la nuit, tu m'appelleras, comme ça, je boirai un verre d'eau avec toi" ". Pour les hommes et les femmes correspondant à ce premier type, le maintien du secret est le plus souvent un choix pour continuer à vivre le plus normalement possible, mais ces dernières sont aussi plus nombreuses à s'y enfermer et à le subir.

Maintenir la vie normale dans la continuité de ce qu'elle était avant le VIH peut s'effectuer aux prix d'arrangements parfois difficiles dans la vie sociale et professionnelle mais se heurte dans la vie intime à l'irrémédiable : la transmission sexuelle du virus. La situation affective et sexuelle des femmes correspondant à ce type est marquée par une grande stabilité : elles sont les plus nombreuses et depuis longtemps à vivre en couple, et presque toutes les femmes en couple de l'enquête s'y retrouvent. Cinq femmes vivaient en couple au moment de la découverte du VIH - dont deux en couples sérocondordants - et le sont toujours au moment de l'entretien plusieurs années après. Cette stabilité affective ne signifie pas pour autant épanouissement sexuel comme l'exprime sans détour Christiane: "Pendant ces deux ans (avant d'apprendre la contamination), on n'a pris aucune précaution particulière, mon mari s'est fait contrôler, il n'a jamais été contaminé [...]. La notion de relation sexuelle est systématiquement associée à la notion de contamination [...]. Pour moi, ce n'est pas un moment de plénitude, dans la mesure où il y a toujours le reste (i.e. le VIH et le risque de contamination). " C'est davantage le corps contaminant et donc dangereux qui est au cœur de la peur exprimée par les femmes que le corps sale et souillé [5, p. 115-116]. Seule Nicole a vu sa relation interrompue par la découverte du VIH : son compagnon a pris peur et a progressivement refusé tous contacts physiques, puis l'a quittée. Comme Nicole, les femmes sont à la fois plus nombreuses et/ou disent plus souvent que les hommes faire l'expérience de la peur de l'autre au niveau sexuel [2, p. 89]. Si le suivi de Nicole entre 1998 et 2000 a montré ses peurs et ses difficultés, il a aussi permis de voir qu'après dix-huit mois de traitement, elle a rencontré un homme à qui elle a pu annoncer son statut sérologique.

Oriane, qui a élevé seule sa fille, « a fait depuis plusieurs années une croix sur sa vie de femme ", alors que Geneviève ressent douloureusement l'absence de relation affective et le fait de ne pas avoir d'enfants. Christiane et son mari ont entrepris une démarche d'adoption, après plusieurs tentatives de grossesse. Mais aucune des femmes correspondant à ce type n'a mentionné un avortement. Si la transmission sexuelle du VIH est une limite objective incontournable pour toutes les personnes infectées, elle prend le sens d'une contrainte insurmontable pour maintenir une "vie normale ", surtout pour les femmes qui n'ont pas d'enfant et/ou qui sont seules. L'adoption de relations sexuelles protégées dans la durée est un véritable défi pour ces femmes qui vivent depuis plusieurs années avec le virus [2]. Elles reconnaissent qu'avec le temps, "elles ont moins envie " et celles qui ont été contaminées par l'homme avec lequel elles vivent toujours "n'ont plus envie ». Les hommes vivant avec le VIH, en revanche, ne font guère état d'une baisse de leur libido.

Dans les premières années du sida, la médecine avait surtout un rôle de surveillance et d'accompagnement des personnes atteintes et ses moyens d'action ont été inexistants jusqu'en 1987, avec les débuts de I'AZT. Comme plusieurs hommes, Jeanne, Geneviève Christiane et Oriane, qui ont appris leur contamination avant 1990, n'ont pas toujours eu un suivi médical régulier et ont privilégié la continuité de la vie et le "faire comme si " sans consulter ou en le faisant irrégulièrement. Ainsi Christiane a interrompu son suivi médical pendant cinq ans et Oriane, qui vit seule avec sa fille, ne s'est pas fait suivre pendant douze ans après son dépistage du VIH en 1985 car dit-elle : « J'ai fait abstraction du VIH et j'ai travaillé comme une folle ”.

Avec l'arrivée des antirétroviraux hautement actifs en 1996, il s'est agi d'apprendre à vivre en même temps avec le VIH et le traitement, comme l'expliquent Nicole et Odile, récemment dépistées et traitées pour la première fois. II faut arriver à « l'intégrer " dans la vie quotidienne et apprendre à vivre avec pour qu'il fasse partie de la vie malgré les changements de molécules, de posologies et de formes galéniques et, surtout, malgré les contraintes qu'il impose et les nombreux inconvénients qui l'accompagnent [9]. Les thérapies antirétrovirales sont devenues une composante essentielle de la vie des personnes atteintes par le $\mathrm{VIH}$, qui vivent accompagnées par la médecine. Elles sont une contrainte incontournable et vitale mais adaptée à leurs conditions de vie et non l'inverse. Contrainte, mais également ressource, qui vient 
s'ajouter aux ressources existantes pour continuer à " vivre le plus normalement possible ".

Si la signification de " la continuité sous contraintes " se maintient avec l'arrivée des traitements, elle connaît un certain infléchissement. Au cours de la période étudiée, un glissement s'est effectué d'une continuité sous contraintes, toute entière du côté de la personne infectée par le VIH/sida, vers une continuité dont la contrainte est en partie aidée et soutenue par la médecine et partagée avec les professionnels. Mais le modèle médical, avec ses références au malade observant et ses valeurs, est maintenu à distance et limité au temps de la consultation et au suivi des prescriptions, sans qu'il y ait pour autant altération de l'image que l'on a de soi et que l'on veut donner de soi.

\section{Discontinuité et retournement}

Ce deuxième type d'expérience est construit autour des questionnements et des remises en cause qui surgissent plus ou moins rapidement à la suite de la découverte de l'infection par le VIH. Les interrogations portent sur les ajustements et les changements d'une vie maintenant incertaine, mais qui se doit d'être néanmoins " normale ". L'infection par le VIH peut alors être considérée comme un déclencheur à l'origine d'une prise de conscience dont les conséquences vont dépendre de l'âge de la personne et de sa situation sociale mais qui, dans tous les cas, conduit à retourner le sens de l'épreuve. C'est un processus plus ou moins long, discontinu et marqué par des bouleversements, qui va déboucher sur de nouveaux choix parfois en rupture avec la vie d'avant. II s'agit alors de savoir " que faire ", face à cette remise en cause de la vie devenue fondamentalement incertaine.

Seule Sophie, qui a découvert sa contamination à l'âge de 22 ans, en 1986, correspond à ce type d'expérience. Cela estil lié au petit nombre de femmes interviewées ou, plus fondamentalement, aux difficultés qu'elles pourraient rencontrer pour retourner le sens de l'épreuve? Une telle remarque n'est pas sans évoquer ce qu'écrit Dominique Schnapper à propos des catégories les plus défavorisées et que l'on pourrait étendre aux plus discriminées: " Les possibilités de retournement du sens chez les plus marginalisés restent pauvres. C'est un pauvre jeu que les pauvres peuvent jouer » [18, p 94].

Sophie est aussi la seule à raconter qu'elle a quitté son précédent compagnon après un avortement, alors qu'elle désirait un enfant et parce qu'il refusait systématiquement le préservatif. " I/ a eu de la chance et moi aussi par la même occasion de passer au travers, c'est vrai que je me suis posée des questions au bout de quatre ans "est-ce que je le transmets" ? [...] C'est vrai que ça peut paraitre complètement aberrant de prendre ce genre de décision, en même temps c'est vrai que je me dis "mais pourquoi je l'ai quitté ?" surtout que j'aurai sûrement mon enfant, mais je l'ai quitté parce qu'il y avait d'autres choses et que dans la vie ce n'est pas seulement avoir un enfant ". Des travaux de sciences sociales ont souligné que les femmes disent plus souvent que les hommes, avoir eu des partenaires qui ont refusé de mettre le préservatif $[2$, p. $97 ; 21$, p. $109-110 ; 22$, p. 12 et 16$]$. Vivant avec un nouveau compagnon depuis plusieurs années, Sophie souffre de son refus d'avoir un enfant. Selon Green ([23, p. 150], le projet d'enfant peut être une source de tension dans les couples sérodifférents, en particulier quand l'homme est infecté par le VIH.

II ne s'agit pas seulement de dissimuler le stigmate ni même de le réinterpréter pour dépasser le discrédit du sida [24], mais plus fondamentalement de retourner le sens de l'expérience et ainsi transformer le temps à vivre. La spécificité de " la discontinuité et du retournement » réside dans l'importance prise par la lutte pour vivre depuis le VIH. Les formes de cette lutte sont diverses, allant d'une lutte personnelle avec le développement d'activités individuelles menées parallèlement à l'emploi salarié, comme dans le cas de Sophie, jusqu'à des formes diverses d'implication et d'engagement dans le monde associatif et la lutte contre le sida, pour plusieurs des hommes interviewés en 1990 et en 1996. Mais le processus de retournement du sens de l'expérience semble s'infléchir depuis l'arrivée des traitements antirétroviraux. La tendance ira-t-elle vers le renforcement des deux autres types?

\section{Enfermement}

Dans ce troisième type, le récit évoque de nombreux événements, sans qu'il soit toujours possible d'identifier le moment de leur survenue. Une chronologie approximative et le brouillage des repères rendent compte d'une histoire marquée de nombreuses ruptures et d'une accumulation d'événements malheureux. La découverte du VIH apparaît comme un malheur supplémentaire qui prend place dans un parcours chaotique et une vie peu stabilisée, sans réel ancrage social et professionnel. Elle peut également être à l'origine de difficultés insurmontables dans une vie fragile et fragilisée. Pour Françoise, l'origine de ses malheurs est clairement identifiée : "Ma vie déjà était chaotique du fait de mon accident de moto à 17 ans. Je ne me suis pas adaptée, je n'ai pas réussi à prendre le dessus, à chaque fois j'ai replongé dans la poudre [...]. Finalement, je n'ai rien construit, je n'ai rien fait, rien n'a avancé dans ma vie. "Cette fatalité de l'accumulation des malheurs conduit à une relative passivité par rapport à une vie dont le sens demeure d'autant plus difficile à (re)trouver que la mort rôde. Fatalité et passivité n'impliquent pas pour autant de ne rien faire pour soi. En effet, l'aide des professionnels est activement recherchée, qu'il s'agisse de médecins ou de travailleurs sociaux: ils sont les béquilles indispensables d'une vie sans réelle attache ni perspective.

Cinq femmes, dont trois qui, ayant perdu leur mari du sida, vivent seules et contaminées depuis plusieurs années au moment de l'entretien, correspondent à ce type. Les quatre femmes contaminées par usage de drogue par voie intraveineuse s'expriment plus volontiers et plus facilement que les hommes sur leurs pratiques 
de consommation et les différents types de drogues. Elles reconnaissent " aimer être cassée » selon leur expression. Green [3, p. 141] remarque que les personnes infectées par injection de drogue maintiennent plus souvent une activité sexuelle, ce qui est le cas pour ces quatre femmes. En revanche, Viviane, infectée par son mari hémophile décédé en 1986, associe blocage sexuel, rapports protégés et diminution des sensations. Mais elle est surtout très préoccupée par l'annonce de sa sérologie qu'elle nomme " la chose " et dont elle est la seule à parler en termes d'aveu " si je fais la connaissance d'un autre gars, déjà je dois lui avouer ma chose. "Si Florence a eu une ligature des trompes après la découverte du VIH, Cécile envisage une grossesse avec l'arrivée des traitements, alors qu'elle évoque rapidement un avortement après la mort de sa fille et de son mari. Un nombre plus élevé de stérilisations et d'avortements a été observé chez les femmes infectées avant 1994 que chez les femmes séronégatives [25].

Ces femmes mènent une vie solitaire et relativement désocialisée. Trois d'entre elles n'élèvent pas leurs enfants qui sont confiés à la famille. Elles se sentent inutiles et désœuvrées et semblent peu soucieuses de l'image qu'elles donnent d'elles et de celle que les autres ont d'elles. Elles sont tributaires et dépendantes de quelques proches comme des professionnels du secteur médical et social. Cette image de soi pour soi, victime enfermée dans ses malheurs, n'est pas pour autant la conséquence du VIH, bien que celui-ci contribue souvent à la renforcer.

Avec les années de vie avec le VIH, les liens sociaux vont perdre de leur intensité et la survenue de chaque événement remet tout en question. Tel est le cas de Jacqueline depuis la mort de sa mère: "J'ai souvent commencé des stages professionnels que j'ai jamais terminés parce que ça ne m'intéressait pas vraiment. Je me rends compte que c'était possible parce que j'avais l'appui de ma mère, toujours [...]. Depuis neuf mois qu'elle est morte, je ne m'en suis pas encore remise, ça a été un choc pour moi. » Cette perte de sens peut s'accompagner de la reprise de conduites addictives pour surmonter le vide qui sera comblé avec plus ou moins de succès par l'aide des professionnels. C'est eux qui fixeront des objectifs et proposeront une organisation du temps avec des visites régulières et donc un véritable encadrement de la vie. Entre 1998 et 2000, malgré un suivi médicosocial régulier et contraignant, Florence a fait une tentative de suicide, a repris régulièrement de la drogue et a été en cure de désintoxication pour l'alcool.

La médecine se trouve ici dans une position centrale, en donnant son sens à la situation présente. Avant 1996, quand ses moyens thérapeutiques sont peu nombreux, elle apporte un soutien et un réconfort à travers une prise en charge psychologique ; depuis l'arrivée des traitements, elle permet de reconnaître l'état de maladie. La médecine devient donc un recours indispensable, qui peut procurer les moyens de vivre en ouvrant des droits et en donnant un statut de malade. Statut qui est moins stigmatisant que celui de droguée, d'alcoolique ou de chômeuse. L'institution médicale, par son pouvoir de sanction, occupe une position centrale pour certaines personnes en situations de précarité.

L'enfermement est le seul des trois types à prendre un sens différent avec l'arrivée des traitements, qui font passer d'une vie vide de sens à une vie dans la médecine. Car le traitement ne suffit pas toujours à la reprise d'un projet de vie quand les ressources sur lesquelles s'appuyer sont limitées. Être traité signifie alors la maladie pour soi et pour les autres et permet de devenir un malade, pris en charge et bénéficiant de droits. Le présent ne peut se vivre qu'avec un étayage social et médical et l'ouverture de droits sociaux. Seul le statut de malade octroyé par la médecine permet de combler la vacuité d'une vie isolée et solitaire. L'accès aux droits sociaux et à l'allocation d'adulte handicapé pourra-t-il se maintenir avec l'évolution des pratiques de reconnaissance du handicap, dans le cadre de la loi de 2005 et avec le plus grand nombre de femmes infectées en situation socio-économique difficile?

\section{Conclusion}

La construction d'une typologie a permis de montrer la diversité de ce que recouvre "une vie normale " pour des personnes qui ont vécu plusieurs années avec le VIH. Des spécificités ont été soulignées dans l'expérience de la vie des femmes. Elles font souvent état de leur isolement dans une maladie longtemps à dominante masculine et sont plus nombreuses à s'enfermer dans le silence et à le subir. Elles ont davantage tendance à vivre leur corps comme contaminant et dangereux lors des rapports sexuels et pour mener à bien leurs projets d'enfant. Ces types d'expérience de la vie avec le VIH, profondément marqués par la participation au monde du travail, la médecine et l'État-providence, résisteront-ils aux changements en cours, marqués par l'augmentation du nombre de femmes infectées par le $\mathrm{VIH} / \mathrm{sida}$ qui sont souvent en situation de précarité économique, avec les transformations du marché du travail, les contraintes financières et l'effritement de l'État-providence?

\section{Références bibliographiques}

1. Weitz R. Powerlessness, invisibility, and the lives of women with HIV disease. In Advances in Medical Sociology, GL. Albrecht GL, RS Zimmerman Eds. Vol. 3, 1993 : The Social and Behavioral Aspects of AIDS, 1993, p. 101-121.

2. Delor F. Vie sexuelle des personnes atteintes, sérodiscordance et risque du sida. In Séropositivité, vie sexuelle et risque de transmission du VIH. Paris: Anrs, 1999, p. 85-100.

3. Green G. Positive Sex: Sexual Relationships Following an HIV-Positive Diagnosis. In AIDS : Foundations for the Future, P Aggleton, P Davies, G Hart, Eds. London : Taylor and Francis, 1994, p. 136-146. 
4. Crawford J, Lawless S, Kippax S. Positive women and Heterosexuality: Problems of Disclosure of serostatus to Sexual Partners. In AIDS : Activism and Alliances, P Aggleton, P Davies, G Hart, Eds. London : Taylor and Francis, 1997, p. 1-14.

5. Théry I. « Une femme comme les autres », séropositivité, sexualité et féminité. In Séropositivité, vie sexuelle et risque de transmission du VIH. Paris : Anrs, 1999, p. 113-136.

6. Ciambrone D. IIIness and other assaults on self : the relative impact of HIV/AIDS on women's lives. Sociology of Health and IIIness $2001 ; 23,4: 517-540$.

7. Doyal L, Anderson J. My fear is to fall in love again... How HIV-positive African women survive in London. Social Science and Medicine $2005 ; 60$ : 17291738.

8. Pierret J. Vivre avec le VIH. Enquête de longue durée auprès des personnes infectées, Paris : PUF, collection "Le lien social $» 2006$.

9. Pierret $J$. Analyse dynamique de la mise sous multithérapie dans la cohorte française Aproco (1998-2000). In Les traitements antirétroviraux. Expériences et défis, J Lévy, J Pierret, G Trottier, Eds. Québec : Presses de I'Université du Québec, Collection "Santé et Société », 2004 : 61-93.

10. Bury M. Chronic illness as a biographical disruption. Sociology of Health and IIIness $1982 ; 4: 167-182$.

11. Bury $M$. The sociology of chronic illness : a review of research and prospects. Sociology of Health and IIIness $1991 ; 13: 451-468$.

12. Charmaz K. Experiencing chronic illness. In: GL Albrecht, R Fitzpatrick, SC Scrimshaw, Eds. The handbook of social studies in health and medicine. London: Sage Publications, 2000, p. 277-92.

13. Conrad P. The experience of illness: Recent and new directions. In Research in the sociology of health care, JA Roth, P Conrad P, Eds: The experience and management of chronic illness. Vol. 6 Greenwich, CT : JAI Press, 1987, p. 1-32.
14. Lawton J. Lay expériences of health and illness : past research and future agendas. Sociology of Health and IIIness $2003 ; 25$, silver anniversary issue : 23-40.

15. Pierret J. The illness experience : state of knowledge and perpectives for research. Sociology of Health and IIIness $2003 ; 25$, silver anniversary issue : 4-22.

16. Locker D, Kaufert J, The breath of life : medical technology and the careers of people with post-respiratory poliomyelitis. Sociology of Health and IIIness $1988 ; 10: 23-40$.

17. Lawton J. Contemporary hospice care: the sequestration of the unbounded body and * dirty dying . Sociology of Health and IIIness $1998 ; 20: 121-143$.

18. Schnapper D, La compréhension sociologique. Démarche de l'analyse typologique. Paris : PUF, collection Le lien social, 1999.

19. Herzlich C, Pierret J, Malades d'hier, malades d'aujourd'hui. De la mort collective au devoir de guérison. Paris : Payot, 1990 (1e édition, 1984).

20. Schnapper D, L'épreuve du chômage. Paris: Gallimard, collection Folio, nouvelle éd, 1994.

21. Meystre-Agustoni G. Prises de risques chez les personnes vivant avec le VIH/ sida. In Séropositivité, vie sexuelle et risque de transmission du VIH. Paris : Anrs, 1999, p. 101-112.

22. Rhodes T, Cusick L, Love and intimacy in relationship risk management : HIV positive people and their sexual partners. Sociology of Health and IIIness $2000 ; 22,1: 1-26$

23. Green G. Sex, Love and Seropositivity : Balancing the Risks. In AIDS: Safety Sexuality and Risks, P Aggleton, P Davies, G Hart, Eds. London : Taylor and Francis, 1995, p. 144-158.

24. Goffman E. Stigmates. Les usages sociaux des handicaps. Paris : Les Éditions de Minuit, 1975, (édition originale, 1963).

25. Lisann Bedimo A, Bessinger R, Kissinger P. Reproductive choices among HIVpositive women. Social Science and Medicine 1998 ; 46, 2 : 171-179. 